\title{
La perspectiva de género en los museos: una tarea pendiente
}

\author{
Rosa Nilda García Osorio \\ Museo Etnográfico y Colonial, Santa Fe \\ UADER-FHAyCS, Argentina (Universidad \\ Autónoma de Entre Ríos. Facultad de \\ Humanidades, Artes y Ciencias Sociales)
}

\section{Resumen}

Las exhibiciones museográficas nos ponen «delante de los ojos» un conjunto de objetos entramados, resignificados, en una trama discursiva y en el marco de un montaje. De este modo, activan memorias, las reinscriben en el presente, las actualizan, pero también las soslayan, las silencian, las minimizan. Desde el año 20II vengo haciéndome eco de muchas autoras y pensando ¿Hay mujeres detrás del «cristal de las vitrinas»? Es decir, ¿estamos representadas en los relatos museográficos? ¿Cómo? ¿Somos agentes de la historia y sus procesos, las mujeres? ¿A qué ámbitos y objetos se asocia la representación femenina? ¿Qué pasa cuando las mujeres estamos ausentes del relato museográfico? La respuesta es simple: sin las mujeres en sus relatos, los museos ofrecen a las/ los visitantes una trama social y genéricamente incompleta. Este trabajo intenta recuperar las distintas acciones curatoriales realizadas en el Museo Etnográfico y
Palabras clave:

Museos, representación, mujeres 
Colonial y en el Museo Histórico Provincial vinculadas a la visibilización de las mujeres — desde la perspectiva de género- en los relatos museográficos.

\section{Abstract}

\section{Gender perspective at museums: a pending}

\section{task}

Museum exhibitions put "before our eyes» a set of objects within a discursive plot and a montage frame. In this way, they activate memories, re-inscribe them in the present, update, also avoid, silence and minimize them. Since 20II I have been echoing many authors and thinking: Are there women behind the showcase? Are we -women-represented in museographical stories? How? Are we agents of history and its processes? What areas and objects is the female representation associated to? What happens when women are absent from the museographical account? The answer is simple: without women in their stories, museums offer visitors an incomplete plot regarding society and gender. This work tries to recover the different curatorial actions carried out in the Ethnographic and Colonial Museum and in the Provincial Historical Museum linked to the visibility of women - from a gender perspective - in the museographical stories.

\section{Resumo}

\section{A perspetiva de gênero nos museus: uma tarefa pendente}

As exposiçóes do museu colocam «diante de nossos olhos» um conjunto de objetos e moldurados e resinificados, em um enredo discursivo e no âmbito de uma montagem. Dessa maneira, ativam memórias, reinscrevem-nas no presente, atualizam-nas, mas também as evitam, silenciam-nas, minimizam-nas. Desde 20II, venho escutando muitas autoras e pensando: Há
Keywords:

museums, representation, women

Palavras chave: Museus, representação, mulheres 
mulheres por trás do «vidro das vitrines»? Ouseja, estamos representados nas histórias museológicas? Como? Somos agentes da história e de seus processos, as mulheres? A que áreas e objetos associa-se a representação feminina? O que acontece quando as mulheres estamos ausentes do relato museógrafo? A resposta é simples: sem as mulheres em suas histórias, os museus oferecem aos visitantes uma trama social e genericamente incompleta. Este trabalho procura recuperar as diferentes açóescuratoriais realizadas no Museu Etnográfico e Colonial e no Museu Histórico Provincial, vinculadas à visibilidade das mulheres -na perspectiva de gênero- nas histórias museológicas.

\section{La cultura, ese campo de batalla}

Toda persona tiene derecho a tomar parte libremente en la vida cultural de la comunidad, a gozar de las artes y a participar del progreso científico y en los beneficios que de él resulten. Toda persona tiene derecho a la protección de los intereses morales y materiales que le correspondan por razón de las producciones científicas, literarias o artísticas de que sea autora. (Artículo 27, Declaración de los Derechos Humanos).

Si bien esto es cierto, consideremos el contexto general y común de todos los obstáculos para la igualdad entre varones y mujeres que denuncia Marta Lamas. Primero, la distinción público-privado, la asociación de lo privado y lo doméstico como ámbitos excluyentes de las mujeres; la consiguiente marginación/subvaloración femenina y de todos los productos de sus trabajos; las escasas posibilidades de acceso — hasta entrado el siglo $\mathrm{xx}$ la formación profesional, su constante abandono del mercado de trabajo en años esenciales del ciclo de vida, su insuficiente formación profesional, la introyección de un modelo único de femineidad (Lamas:I996) asociado a la maternidad y la ideología de la domesticidad (Pollok: 2013). El campo de la cultura, no escapa a estas determinantes generales y como espacio expresivo, comunicativo, de realización humana, ha excluido completa y/o parcialmente a las mujeres, asignándoles un lugar marginal, subsidiario; como musas o alumnas.

En Argentina, por ejemplo, las colecciones artísticas que conforman el 
patrimonio de los grandes museos, fueron aportadas — desde fines del siglo XIX y principios del siglo XX-, en su mayoría, por varones. Recién en la segunda mitad del siglo pasado se destacan las coleccionistas María Luisa Bemberg, Sara Facio y Nelly Arrieta de Blaquier (Alvarez y Alvarez: 20I6). Pues justamente de la mano de movimientos sociales afirmativos de la condición femenina, cada vez más mujeres exigieron el reconocimiento a su rol protagónico en la producción y el consumo de bienes culturales. Sin embargo, hay que hacer una distinción entre el lugar de productoras de cultura — que siempre han tenido- y el lugar de reconocimiento, visibilización, participación y acceso a los espacios legitimados de la producción cultural, a las industrias culturales. En este sentido, crear y difundir lo creado constituye un obstáculo para todas las mujeres que quieren incidir en las industrias culturales.

\section{Patrimonio, género y patriarcado}

(...) Las mujeres han emergido como sujeto político en la resignificación de los hechos, o en la significación de aquellos hechos silenciados. De significadas han pasado a ser significantes. Solo es una contradicción aparente que las mujeres sean objeto precisamente en el momento en que se convierten en sujeto, cuando dejan de asumir su existencia como un dato y empiezan a interrogarse sobre las condiciones de la misma, en tanto que colectivo unido por una forma común de opresión. Ese ponerle palabras a la vida es sacarla del ámbito de la naturaleza y meterla en la sociedad.

María Jesús Izquierdo

La propia etimología de la palabra patrimonio deviene del latín patri ('padre') y monium ('recibido'), que significa «lo recibido por línea paterna». Jimenez Esquinas (20I6) señala que en el origen se incluye una falta de neutralidad y un vínculo con el patriarcado -gobierno o autoridad de los patriarcas- que es difícil de obviar. En los años ‘ 60 , al calor de los movimientos de liberación femenina, el feminismo como movimiento político comenzó a pregnar todas las instancias sociales generando institucionalmente las condiciones para que la desigualdad pueda ser desnaturalizada, desencializada y pensada científicamente (Lamas: 1996:17). Esto posibilitó combatir el determinismo biológico como paradigma hegemónico, evidenciando las desigualdades y legitimó la necesidad de intervenir y transformar. Se abrió así un cuestionamiento radical de los contenidos, la organización, e incluso, la forma de conocimiento de los marcos teóricos vigentes, institucionales o no. Saber y crear son, de hecho, cuestiones políticas: "(...) la invisibilidad de las mujeres no sucede porque no existan, ni porque no conozcamos sus nombres. No es tampoco porque ellas no tengan importancia 
como artistas creadoras de la cultura de la época moderna. Se produce porque las estructuras de nuestro saber son de hecho androcéntricas» (Pollock: 20I3).

En los ámbitos de la cultura y el patrimonio, las mujeres han ido paulatinamente jugando un rol fundamental en la difusión del patrimonio, el arte, los estudios históricos y arqueológicos. Desde los años '9o, la crítica de género viene denunciando que el patrimonio no es neutral. Lo atraviesan importantes sesgos que contribuyen a la legitimación, el refuerzo y la perpetuación de estereotipos androcéntricos y patriarcales que naturalizan las desigualdades entre varones y mujeres. Susana Rostagnol (20I5) señala: «Las prácticas y los conocimientos de las mujeres —y más aún si se trata de mujeres indígenas- están invisibilizados al no recibir reconocimiento por parte de sus comunidades. No son prestigiosos. Muy probablemente esta valoración sea compartida por la comunidad en su conjunto. Los ejemplos de conocimientos y prácticas que carecen de reconocimiento son múltiples; van desde canciones de cuna, dichos, técnicas relativas a trabajos manuales, cocina, remedios. Es muy claro como en la cultura del medio rural ganadero goza de más prestigio el trabajo del huasquero (masculino) que el de las mujeres para confeccionar las jergas (también llamadas matras) que forman parte del apero. La no valorización de los remedios caseros en base al uso de yuyos, conocimiento que en general se encuentra entre las mujeres ancianas, es compartida por toda la comunidad, es posible que esté asociado al valor positivo de lo moderno (tratamientos médicos y remedios farmacológicos en este caso) en detrimento de los conocimientos tradicionales». Es necesario generizar el patrimonio (Saprisa y Via Cherro:20I6), evidenciar la desigual valoración de los aportes culturales de varones y mujeres, problematizar los mandatos atribuidos según las condiciones sexo-genéricas y señalar que la excepcionalidad — sin bien debemos reconocerla- contribuye a fortalecer la idea de que es una excepcionalidad que también refuerza el mandato, en tanto surge como tal en términos de desvío con respecto a estos lugares socialmente asignados.

Actualmente se han producido importantes fisuras en los paradigmas que legitimaban «científicamente» tales desigualdades. Esta deslegitimación de la desigualdad social - y no natural de los géneros problematiza, toda la vida cotidiana, los marcos conceptuales y todos los espacios sociales que habitamos, donde se han pensado la feminidad y la masculinidad y consiguientemente las relaciones de poder entre géneros. Lo que antes era obvio, hoy ha dejado de serlo.

Las epistemologías tradicionales excluían sistemáticamente la posibilidad 
de que las mujeres y las identidades sexuales subalternizadas fueran sujetas o agentes del conocimiento. Estas lecturas y relecturas nos han permitido repensar el modo en que la ciencia se ha escrito desde el punto de vista heteropatriarcal y eurocéntrico. Necesitamos epistemologías alternativas que nos legitimen a las mujeres como productoras de conocimiento. La perspectiva de género se ha convertido en una pauta de organización tanto simbólica como material de las sociedades, y todos los campos del saber fueron puestos bajo la crítica feminista, elucidando así los sesgos andro y eurocéntricos presentes y activos en la ciencia. Teórica y empíricamente la perspectiva del género como un campo de relaciones de poder nos lleva a analizar el parentesco, la sexualidad, la reproducción, el matrimonio, la filiación y la herencia; las solidaridades, sociabilidades, los conflictos y sus formas de resolución inter e intrafamiliares, tanto en los ámbitos domésticos como públicos. La división social y sexual del trabajo según los géneros, las relaciones de subordinación-dominación entre los géneros analizando cómo se estructura y se ejerce el poder en los distintos ámbitos.

\section{Mujeres y museos: el derecho a la representación}

Mirar el pasado solo debe ser un medio
para entender con mayor claridad qué y
quiénes somos, para poder construir de
forma más inteligente el futuro. Paulo Freire

Como contexto general, nuestro país, Argentina, posee una importante normativa progresista en el campo de los derechos de las mujeres, que ha consagrado todo un andamiaje jurídico (26485, 26150, 26743, 26618, 27499) e incluye la creación de instituciones específicas que bregan por los derechos de las mujeres como el Instituto Nacional de las mujeres (INA), en 20I6. Sin embargo, todavía no podemos decir que lo que en materia de legislación nacional (Ley 26485) ${ }^{1}$ se recomienda: «(...) la eliminación de la discriminación entre mujeres y varones en todos los órdenes de la vida» (art. 2, inc.a); (...) la remoción de patrones socioculturales que promueven y sostienen la desigualdad de género y las relaciones de poder sobre las mujeres» (art. 2 inc.e), sea una realidad.

En el campo de las ciencias sociales y en muchos ámbitos de la producción

1 Ley de Protección Integral para Prevenir, Sancionar y Erradicar la Violencia contra las Mujeres en Ios Ámbitos en que desarrollen sus Relaciones Interpersonales. La Ley, promulgada en abril de 2009 , aspira a eliminar la discriminación entre mujeres y varones en todos los órdenes de la vida, afirmando en particular el derecho de las mujeres a una vida sin violencia. 
científica, artística y cultural se ha consolidado un campo de investigaciones vinculadas a la historia de las mujeres y de las relaciones de género, en infinidad de libros y artículos, jornadas, encuentros y congresos. Sin embargo, en el ámbito de los museos esta es todavía una reflexión marginal y un campo de estudios en construcción. Todavía la perspectiva de género tiene un impacto escaso o nulo en las propuestas curatoriales, educativas y museográficas. Lamentablemente, su inclusión queda en manos de la buena voluntad y el compromiso de las personas (en general, mujeres) que gestionan/trabajan estos espacios. Aunque se proponen muestras y exposiciones sobre el rol de las mujeres y las relaciones de género a través de la historia, la perspectiva de género está lejos de instalarse como un espacio fecundo de investigación y producción cultural orientado al desarrollo de conocimientos y propuestas que posibiliten incluir esta mirada al momento de renovar y replantear las muestras permanentes.

Como otras instituciones de la cultura, los museos son espacios de producción y consumo cultural, y contribuyen a la conformación de identidades e imaginarios colectivos y diversos: étnicos, nacionales, y también genéricos. Los museos y espacios patrimoniales son instancias socializadoras que inciden en la construcción y formación de identidades, subjetividades, imaginarios y memorias colectivas inclusivas, sino pueden/deben contribuir a crear otras formas de aprendizaje desde la igualdad. Se hace necesario, por consiguiente, introducir en estos espacios la perspectiva de género, pues es una herramienta crítica, analítica y reflexiva.

\section{El relato museográfico: una trama social y genéricamente incompleta}

Las exhibiciones, al seleccionar sus temas, expresan valores y creencias, involucran un diseño y códigos particulares, así como la realización de ciertas actividades siempre en incidencia con la cultura a la que están dirigidas. A través de los objetos y los modos en que la exposición los presenta se tejen historias, se evocan sujetos, se explican procesos materiales y significan procesos simbólicos, se pueden restituir sentidos.

En términos generales, la propuesta museográfica del Etnográfico vigente hasta 2018 (hoy en proceso de renovación museográfica, tras el cierre del museo en 20I8) sesgaba la representación femenina, pues era una museografía pensada varios años atrás, que no había tenido innovaciones significativas en cuanto a la perspectiva de género (entre las determinantes más importantes, por falta de presupuesto para una nueva). Entre otras determinantes institucionales, no existía un trabajo colectivo que permitiera pensar desde la perspectiva de género la «ausencia» de las mujeres, y en muchos casos la escasez de fuentes, justificaba la ausencia de las mujeres en la representación. No obstante, 
estas problemáticas no se problematizaban, y las dificultades que entrañaba la ausencia, tampoco. No se ponía en discusión el carácter de los/las usuarios/as o productores/productoras de los objetos expuestos y su condición genérica y social. Desde las cartelas y textos que orientaban a los/las visitantes no se interpelaban las visiones e imaginarios acerca de los ámbitos femeninos y/o la participación de las mujeres en las actividades cotidianas, permitiendo que ellos/as construyeran sentidos e interpretaran, supliendo la falta de información con su sentido común generalizado. Se desplegaban, así, las significaciones construidas en la propia experiencia social, vital, sobre los roles y actividades femeninas, sin mediaciones críticas. Cuestión que nos pone delante de la permanencia y reproducción de miradas sesgadas, estereotipadas y prejuicios acerca de la representación de las relaciones de género y de las mujeres, en particular.

La representación de los ámbitos con presencia de mujeres (por ej. el estrado, en la sociedad colonial) se asume como natural. Es decir, no se indaga acerca del por qué, para qué las mujeres eran asociadas a esos espacios y con ellos a determinadas actividades. Se naturaliza el orden de género vigente. Aunque para otro espacio colonial, López Soto (2018:7) señala que «(...) el estrado femenino no tiene que ser necesariamente un lugar de exclusión y separación entre hombres superiores y mujeres inferiores, sino un contexto de independencia y de gobierno femenino autónomo. En este lugar las mujeres pasaban la mayor parte del tiempo doméstico y contrario a los estudios y bibliotecas masculinos, una casa podía contar con tres o más estrados. Adicionalmente, los estrados podían a su vez acompañar las oficinas masculinas, dividiendo las salas en dos por medio de biombos, conquistando más metros de un 'cuarto' de la casa colonial santafereña».

La participación de las mujeres en los distintos espacios (de sociabilidad doméstica o pública) no aparecen problematizadas, y las dificultades para hacerlo tampoco se abordan. López Soto insiste en la necesidad de revisitar la condición de las mujeres en los espacios coloniales, a los fines de optar por una mirada «retirada», pues desde el alejamiento es posible vislumbrar más autonomía y posibilidades de la condición femenina que las que solemos atribuirle a la Colonia (2018:3). La autora señala la necesidad de problematizar los espacios y ámbitos dentro de la casa, a los fines de considerar el modo en que reforzaban la condición de hija, esposa y/o madre, y agrega que la investigación sobre el género como otra clave de organización de los espacios permite comprender la dinámica familiar, y el funcionamiento social, jerárquicamente atravesado por distinciones de raza, sexo y estatus.

En nuestro museo, las mujeres no están enunciadas como parte del relato 
histórico, menos aún como «agentes» dentro de la trama; a lo sumo, se agrega en las visitas mediadas su carácter de propietarias y/o usuarias de los objetos. Tampoco se hace referencia a la enorme diversidad étnica, social, y etaria de las mujeres, a su carácter de solteras, casadas, viudas, concubinas, etc. Se soslaya toda esta rica información, ofreciendo al visitante un grupo poblacional homogéneo, carente de diferencias y conflictos en su interior. De modo que todo este conjunto de significaciones que podrían desglosarse queda trunca, y la representación de las mujeres —además de sesgada — se piensa ahistórica e intemporal. En paralelo, se ofrece una versión de la historia de las mujeres que no discutieron su destino "femenino", ni transgredieron mandatos. Cabe considerar —además - como afirman Birriel Salcedo, M. y Risquez Cuenca, C. (20r6) «En el desarrollo de la cadena lógica básica (investigar, proteger, conservar y difundir), se reflejan aquellos valores que «se han seleccionado para ser transmitidos», por lo que el resultado final no es neutro, sino producto de una elección. Por otro lado, los objetos no son interpretados e interpelados contextual y críticamente, evidenciando los diferentes contextos que los atraviesan y cómo en todos ellos se sesga a las mujeres:

- histórico/arqueológico, vinculado a la producción, el uso, el consumo y el descarte de artefactos;
- el epistemológico y disciplinar que aborda los problemas científicos, metodológicos, epistemológicos y disciplinares en general de las investigaciones sociales;

- el antropológico-cultural respecto de los sentidos/significados simbólicos y materiales desplegados en esos objetos por las distintas sociedades;

- el museográfico y didáctico, que los resitúa en un relato institucionalmente construido desde la contemporaneidad.

Consideramos que en la puesta museográfica vigente hasta el año 2018 estaba omitida la problematización acerca de las relaciones inter e intragenéricas, contribuyendo a la invisibilización de las mujeres y a su exclusión del ámbito de la representación museográfica y de la representación simbólica de los colectivos femeninos en la reconstrucción histórica que el museo proponía a sus visitantes. En orden a construir explicaciones del porqué de estas ausencias, es necesario considerar también que en estas omisiones se imbrican y operan distintos factores: las limitaciones del registro arqueológico; la ausencia de la reflexión con perspectiva de género en los espacios académicos tanto en la formación como en la construcción de un perfil profesional dentro de las disciplinas sociales; el estado del campo en relación con los trabajos de arqueología de género y su desarrollo para la zona del Litoral; 
entre otros. Pues entre el discurso y la representación museográfica, median las investigaciones provenientes de las áreas científicas específicas de las ciencias sociales: historia, arqueología, antropología. De modo que el relato que el museo propone a sus visitantes se sustenta en los resultados de ellas, y evidenciando sus distancias y proximidades, sus entredichos y sus consensos. Es decir: actuando conjuntamente con las limitaciones propias del registro arqueológico, existen sesgos y prejuicios antropocéntricos presentes en las diversas ciencias sociales que abordaron teórica y metodológicamente el problema. Estos silencios, ausencias y omisiones instituyen un obstáculo epistemológico para la reconstrucción de ese pasado lejano, pues al no ser objeto de la reflexión científica, las mujeres quedan al margen de esa historia.

Acciones curatoriales para restituir memorias e historias de las mujeres
Asistimos a un proceso de profundo cuestionamiento de las representaciones genéricas en general, de los mandatos femeninos en particular. En este marco, las condiciones concretas en las que la desigualdad e inequidad de los géneros se expresan, son profundamente interpeladas: los espacios museográficos no deberían estar ajenos a estas reflexiones que surcan nuestra contemporaneidad.

En el año 20I4 la Organización de las Naciones Unidas para la Educación, la Ciencia y la Cultura (UNESCo) publicó su Reporton Gender Equality and Culture, un conjunto de ocho principios para garantizar la igualdad de género en los ámbitos del patrimonio, la creatividad y la diversidad cultural, es una de las prioridades esenciales de la unEsco. La igualdad de participación, acceso y contribución a la vida cultural de las mujeres y los hombres es un derecho humano, además de un derecho cultural. $^{2}$

2 1. Garantizar la plena aplicación de las convenciones y declaraciones internacionales en el ámbito de la cultura en consonancia con otros instrumentos de derechos humanos y con respecto a la igualdad y la diversidad de género a fin de ampliar los horizontes creativos de mujeres y hombres, niños y niñas, y garantizar la igualdad acceso y participación en la vida cultural.2. Fortalecer la base de evidencia a través de la recopilación y difusión periódica y sistemática por parte de las oficinas nacionales de estadísticas de datos desagregados por sexo en todas las áreas del sector cultural: incluyendo empleo, educación, desarrollo de capacidades, participación y consumo.3. Desarrollar y aplicar políticas y estrategias con perspectiva de género en la cultura que empoderen a todos los miembros de la sociedad, teniendo en cuenta la diversidad de diferentes grupos e individuos, y la intersección de factores sociales más amplios y desigualdades que pueden agravar las desventajas.4. Reforzar las capacidades institucionales nacionales para promover la igualdad de acceso de mujeres y hombres a los procesos de toma de decisiones, recursos financieros y educación en campos culturales. 5 . Establecer iniciativas de liderazgo y tutoría para mujeres creadoras y profesionales del patrimonio y garantizar el equilibrio de género en los niveles superiores de liderazgo en el sector cultural y creativo. $\rightarrow$ 
En general, como trabajadora de un espacio cultural de la provincia de Santa Fe he iniciado un proceso lento, pero sostenido y empecinado para repensar y deconstruir significados, prácticas, representaciones, símbolos y normas socialmente construidos sobre diferencia sexual interpretada en clave de desigualdad en el ámbito de los museos y espacios culturales. Estas reflexiones permitieron ir desarrollando distintas intervenciones: cartelería, curaduría de muestras temporarias, visitas mediadas, generación de contenidos para las redes sociales.

En 2012 se inauguró una exposición curatorial sobre la representación femenina y la construcción de la feminidad en términos históricos, denominada (H)ay! Mujeres ${ }^{3}$. El objetivo era ofrecer una reconstrucción historiográfica de la presencia femenina desde la conquista, incluyendo la formación de los estados nacionales, y el surgimiento del movimiento feminista del siglo $\mathrm{xx}$. Nos preocupaba dejar claro el modo en que la producción teórica se nutre y se «reinventa» en relación con los movimientos sociales y la militancia. A partir de 2013 la muestra fue itinerante, y para acompañar la tarea pedagógica en las escuelas e instituciones que la solicitaban se realizó un material teórico-pedagógico. ${ }^{4}$ En el año 2017 iniciamos la Serie Pioneras. Un conjunto de publicaciones en nuestras redes, recuperando el trabajo y las biografías de las primeras arqueólogas, nos permitimos compartir algunas interrogaciones y algunas búsquedas sobre sus investigaciones, y sus obras. Dimos cuenta de sus aportes al campo de la arqueología en general y, más específicamente, a la arqueología de género. Revisitamos la obra de Elsie Lewis Parsons, Ruth Landes, Phillis Kaberry, Audrey Richards, Margareth Mead... y particularmente a Amelia Larguía

6. Apoyar campañas internacionales, nacionales y locales de sensibilización y promoción que aborden los estereotipos de género y la discriminación en todos los aspectos de la vida cultural. 7. Alentar e involucrar a todos los miembros de la sociedad en estrategias que promuevan la igualdad de género en la cultura. Esto incluye trabajar en asociación con todos los grupos y comunidades interesadas para promover soluciones sostenibles para el acceso, la participación y la contribución a la cultura con igualdad de género. 8. Apoyar la investigación interdisciplinaria sobre la igualdad de género en el patrimonio y las industrias creativas que involucran a grupos y comunidades interesadas, y considerar la complejidad y diversidad de las relaciones de género y las estructuras de poder subyacentes.

3 Se puede descargar gratuitamente de nuestra página web, http://museojuandegaray.gob.ar/ novedad-serv-edu/8/el-museo-va-a-a-escuela.

4 Los materiales producidos han tratado de expresar nuestra convicción acerca del modo en que sobre las diferencias entre los géneros se han construido desigualdades, resultantes del orden social patriarcal. 
de Crouzeilles, infatigable arqueóloga amateur de nuestra provincia. ${ }^{5}$

También en 20I7, participamos institucionalmente de la convocatoria internacional Museum Weeck, donde a lo largo de una semana los museos producen contenidos desde sus redes sociales, en nuestro caso, las temática fueron las mujeres y los estudios de género.

Como trabajadoras del campo de la cultura, adherimos a los paros internaciones de mujeres 2017 y 20I8, realizando actividades en nuestra sala de exposiciones.

Personalmente propuse y realicé visitas mediadas en el Museo Histórico Provincial: «Entre olvidos y silencios. Mujeres santafesinas en el siglo XIX» (30/04/19). Una propuesta dirigida a pensar las relaciones de género y étnicas en el marco de nuestra historia local, poniendo énfasis en la situación diferencial del colectivo de mujeres de la sociedad colonial intersectando género, etnia y clase social. En la misma institución se presentó el libro «Mujeres en tránsito. Viajes, identidad y escritura en Sudamérica» (4/o7/19), junto a su autora, la Doctora Vanesa Miseres, con los comentarios de Natalia Zendher (psicóloga) e Ileana Sáenz (politóloga), quienes junto a la autora reflexionaron sobre el viaje y la escritura como procesos que impactan psíquica y socialmente en la condición femenina.
En el Museo Histórico, también, durante todo el mes de junio se desarrolló el Curso/conversatorio: «Mujeres de armas toman». Destinado a pensar la agencia femenina en los procesos revolucionarios del siglo xIx en el litoral rioplatense. Esta fue una instancia pensada para generar un espacio de conversación, socialización de bibliografías y reflexión, al que asistieron más de 90 personas.

El último de los proyectos es Mujeres de dos orillas. Esta muestra irá acompañada de un dispositivo para el desarrollo de un recorrido autoguiado, propuestas de guiones para la elaboración de visitas guiadas y una publicación on line en los sitios de cada museo para el trabajo en el aula de las escuelas primarias y secundarias de ambas ciudades. Este proyecto propone la participación coordinada dos instituciones: el Museo Etnográfico y Colonial Juan de Garay, dependiente del Ministerio de Innovación y Cultura de la Provincia de Santa Fe y el Museo del Cabildo de Montevideo, dependiente del Ministerio de Cultura de la ciudad de Montevideo.

Finalmente durante 2019, se llevó adelante un proyecto ideado por Analía Molinari (Equipo Educativo del Museo Histórico Provincial), del que participé de julio a octubre. Denominado «La pie$z a$ del mes» el proyecto presentó durante

5 Teresa Suárez, publica un artículo sobre la biografía de Amelia en Historiografía y sociedad. Discursos, instituciones e identidades. 2010. Ediciones UNL. 
todo el año, historias, biografías, interrogantes, y reflexiones históricas acerca de las mujeres en Santa Fe, utilizando como fuentes las fotografías del archivo del Museo Histórico Provincial. En diciembre, se realizará la presentación de todo el trabajo mediante un panel, donde invitamos a trabajadoras de otros museos santafesinos a conversar y compartir sobre las intervenciones museográficas que apunten a visibilizar a las mujeres.

\section{La necesidad de pensar los museos desde una perspectiva de género y decolonial}

En la realización de todas estas actividades tuvimos como objetivos presentar categorías para comprender la construcción histórica de la feminidad y la consecuente invisibilización femenina en la historia y las ciencias sociales, criticarla y trascenderla, contribuir al enorme proceso de movilización social del movimiento feminista aportando herramientas teóricas para fundar posiciones y debates que impulsen el cambio social, e incorporar las perspectivas epistemológicas alternativas respecto de la decolonialidad, feminismo e interculturalidad en el trabajo crítico de las ciencias sociales y los museos. De conjunto todas estas actividades tuvieron por objetivo restituir distintas aspectos de la presencia, el accionar, la agencia femenina en nuestra historia local, contextualizar desde una perspectiva de género objetos y usuarios/as, poner en tensión los relatos museográficos señalando sus ausencias, indicando lo urgente y necesario que resulta abrir camino a estas reflexiones.

El género constituye hoy un dispositivo teórico que nos permite deconstruir significados, prácticas, representaciones, símbolos, instituciones y normas socialmente construidos sobre diferencia sexual interpretada en clave de desigualdad. Si deseamos comprender cabalmente la profundidad y extensión de las transformaciones que requiere un mundo diverso y plural, debemos reconocer la complejidad de articulaciones entre géneros, etnias y clases que producen sujetos y prácticas no homogéneos. Se trata de aspirar a la igualdad, respetando las diferencias, bregando por eliminar la parcialidad de las miradas androcéntricas y las distorsiones que el sexismo y el patriarcado producen.

La perspectiva de género en los museos viene a garantizar y promocionar derechos. A abrir espacios de igualdad en la representación, la construcción, el acceso y el disfrute del patrimonio. Adoptar esta perspectiva no solo hará visibles a las mujeres en el pasado — valorando su legado- sino que también incidirá en el empoderamiento de las mujeres y niñas de hoy y mañana.

Para calibrar el impacto de este cambio de perspectiva pensemos ¿Cómo nos sentimos hoy las mujeres y las nińas que vamos a los museos y no estamos consideradas, 
reflejadas, valoradas en sus relatos? ¿Cómo lidiamos con los prejuicios, los supuestos y los estereotipos presentes en las exposiciones temporales? ¿Cómo hacemos para entender y asumir que sí somos parte de la historia? ¿Nos acostumbramos a la injusticia, a la desigualdad? ¿ No aprendemos — también allí- a ser menores, prescindibles, poca cosa?

No olvidemos. No existen museos neutrales ni formas asépticas e inocentes de representar el pasado y sus protagonistas. Los museos y espacios patrimo- niales intervienen significativamente en la construcción, producción, difusión/ circulación, acceso y apropiación del conocimiento y la cultura social. Recuperar la memoria de las mujeres nos permite cuestionar la desigualdad que vivieron y viven, colaborado en la erradicación de patrones discriminatorios, sexistas y misóginos, que reproducen las violencias contra las mujeres. Las instituciones del estado son responsables de esta tarea, y dentro de ellas, los museos no pueden permanecer indiferentes.

Bibliografía:

- ALVAREZ, Ángeles y ALVAREZ GUTIÉRREZ, Marta Historias de argentinas. Mujeres argentinas en la historia de los últimos doscientos años. 2016. En La memoria femenina. Mujeres en la historia, historia de mujeres. Patrimonio en Femenino. Ministerio de Educación, Cultura y Deporte-lbermuseos.

- ALVA MENDOZA, Bessna Raquel (2008). De mujeres, museos y monederos. Mujeres por el derecho al disfrute de las artes, Revista Decisiones, № 20.

- BIRRIEL SALCEDO, Margarita y RÍZQUEZ CUENCA, Carmen. (2016) Patrimonio, turismo y género. Estrategias para integrar la perspectiva de género en el patrimonio histórico. Perspectivas. Revista $P H$ Instituto Andaluz del Patrimonio Histórico No 89 pp. 126-127.

- CARREÑo ROBLES, Eva. (2016) Museos en clave de género. Revista PH No 89. Pg. 156-157.

- CASTILLA, Américo (2010) El museo en escena. Política y cultura en América Latina, México: Paidós.

- COCCO, Gabriel y FEUILLET TERZAGHI, María del Rosario (2010) (Comps.) Arqueología de cazadores recolectores en la Cuenca del Plata. Revista América. Centro de Estudios Hispanoamericanos, Santa Fe. 
- CORDERO REIMAN, Karen y SÁENZ, Inda (Comps.) (S/F) Crítica feminista en la teoría de la historia y el arte. Universidad Iberoamericana, Ciudad de México, Programa de Universidad y Género.

- DE BARBIERI, Teresita (1993) Sobre la categoría género. Una introducción metodológica. Debates en Sociología. UNAM.

- DEVOTO, F. y MADERO, Marta (1999). Historia de la vida privada en Argentina. La Argentina Plural. Buenos Aires: Taurus.

- JIMÉNEZ-ESQUINAS, Guadalupe: (2016) De «añadir mujeres y agitar" a la despatriarcalización del patrimonio: la crítica patrimonial feminista, Perspectivas. Revista PH Instituto Andaluz del Patrimonio Histórico No 89 pp. 137-140.

- LAMAS, Marta La perspectiva de género. (En línea) Consultado el 09/10/19 en https://www.ses.unam.mx/curso2007/pdf/ genero_perspectiva.pdf

- LAMAS, Marta(1996) El género: La construcción cultural de la diferencia sexual. México: Miguel Ángel Porrúa. Librero Editor.

- LAGUNAS, Cecilia y Ramos, Mariano (1997) Patrimonio y cultura de mujeres. Jerarquías y espacios de género en museos locales y en institutos oficiales nacionales, Revista La Aljaba, Segunda Época, Volumen XI.

- LÓPEZ SOTO, María Marcela (2018). Enseres del estrado santafereño: otra mirada a la autonomía femenina en el Nuevo Reino de Granada. (En línea, consultado el 10/10/19 en: https://www.academia.edu/36651588/Enseres_del_estrado_ santafere\%C3\%B10_otra_mirada_a_la_autonom\%C3\%ADa_femenina_en_el_Nuevo_Reino_de_Granada

- MACEIRA OCHOA, Luz (2008) Género y consumo cultural en museos. Análisis y perspectivas, en Revista La Ventana $N^{\circ} 27$. - MC PAHILFANGER, Elsie (2008) Mujeres (ad)miradas y mujeres que miran, en Revista La Ventana, $\mathrm{N}^{\circ} 28$.

- POLLOCK, Griselda (2013) Visión y diferencia. Feminismo, feminidad e historias del arte. Buenos Aires: Fiordo Editorial.

- ROSTAGNOL, Susana (2015). ¿El patrimonio tiene género? Una mirada al patrimonio cultural inmaterial desde la perspectiva de género. En Primer Encuentro Nacional de Patrimonio vivo. 
Diversidad cultural y estado: Escenarios y desafíos de hoy. CCK. (En línea) Consultado el 16/11/19 en https://www.academia. edu/20380032/_El_patrimonio_tiene_g\%C3\%A9nero_ Una_mirada_al_patrimonio_cultural_inmaterial_desde_la_ perspectiva_de_g\%C3\%A9nero

- ROTKER, Susana (1999) Cautivas. Olvidos y memorias en la Argentina, Buenos Aires: Ariel.

- SAPRISA, Graciela y VIERA CHERRO, Mariana (2016) Generizar el patrimonio. Algo más que objetos creados por mujeres, en: En La memoria femenina. Mujeres en la historia, historia de mujeres. Patrimonio en Femenino. Ministerio de Educación, Cultura y Deporte - Ibermuseos. Publicación electrónica. Disponible en http://www.ibermuseos.org/wp-content/uploads/2016/03/LAMEMORIA-FEMENINA-PUBLICACION-ELECTRONICA.pdf 